\title{
Efecto del consumo de endulzantes en la ingesta de energía y tejido adiposo: una revisión
}

\author{
Effect of the consumption of sweeteners on energy intake and adipose tissue: \\ a review \\ Jocelyn Gómez Castillo ${ }^{a}$, Guadalupe López-Rodríguez, Jonathan Alvarez ${ }^{c}$, Shirley Dominig \\ Aguilera Chávez ${ }^{d}$
}

\begin{abstract}
:
Mexico is one of the countries with high consumption of sugary drinks, 163 liters per capita per year. Overweight and obesity are a public health problem in Mexico, which coincides with the increase in the consumption of sweetened beverages. In animal and human models, the intake of beverages with caloric and non-caloric sweeteners is associated with a loss in regulation of energy intake and the accumulation of adipose tissue (AT). It has been described that chloric and non-caloric sweeteners can affect weight, triglyceride concentrations and promote the accumulation of AT, however, there is no conclusive evidence on the metabolic and adiposity effect after chronic consumption of caloric and non-caloric sweeteners. This review aims to present the scientific evidence available in scientific papers on the effect of sweetened beverages in relation to energy intake and the accumulation of adipose tissue.
\end{abstract}

Keywords:

Caloric sweeteners, non-caloric sweeteners, energy intake, body weight, adipose tissue

\section{Resumen:}

México es uno de los países con alto consumo de bebidas azucaradas, 163 litros per cápita al año. El sobrepeso y la obesidad son un problema de salud pública en México, lo cual coincide con el aumento del consumo de bebidas endulzadas. En modelos animales y humanos, la ingesta de bebidas con endulzantes calóricos y no calóricos está asociada con la pérdida en la regulación de la ingesta de energía y la acumulación del tejido adiposo (TA). Se ha descrito que los endulzantes calóricos y no calóricos pueden afectar el peso, concentraciones de triglicéridos y favorecer la acumulación de TA, sin embargo, no existe evidencia concluyente sobre el efecto metabólico y de adiposidad tras un consumo crónico de endulzantes calóricos y no calóricos. Esta revisión tiene el objetivo de presentar la evidencia científica disponible en artículos científicos sobre el efecto que tienen las bebidas endulzadas con relación a la ingesta de energía y la acumulación del tejido adiposo.

\section{Palabras Clave:}

Endulzantes calóricos, endulzantes no calóricos, ingesta de energía, peso corporal, tejido adiposo

\section{Introducción}

Los seres humanos tienen una predilección hacia los alimentos dulces. Sin embargo, su consumo se ha asociado al desarrollo de enfermedades crónicas como la obesidad y la diabetes. Antes de la Segunda Guerra Mundial se utilizaban sólo endulzantes calóricos, pero durante la guerra hubo una escasez importante de azúcar y un cambio de la estética a favor de una figura delgada, que animó a las mujeres a recurrir a sustitutos artificiales. ${ }^{1}$ Los sustitutos de azúcar están divididos en 2 grandes grupos: endulzantes calóricos (EC) y endulzantes no calóricos (ENC). ${ }^{1}$ Los ENC se utilizan principalmente por la industria de alimentos para sustituir total o parcialmente el azúcar (sacarosa), ya que poseen un mayor poder

\footnotetext{
Autor de Correspondencia, Universidad Autónoma del Estado de Hidalgo, https://orcid.org/0000-0001-6727-0601, Email: go230170@uaeh.edu.mx

${ }^{\mathrm{b}}$ Universidad Autónoma del Estado de Hidalgo, https://orcid.org/0000-0001-5432-0382, Email: glopez@uaeh.edu.mx

c Universidad Autónoma del Estado de Hidalgo, https://orcid.org/0000-0002-9434-997X, Email: becarioacademiasnutricion@gmail.com

d Universidad Autónoma del Estado de Hidalgo, https://orcid.org/0000-0002-5729-4797, Email: ag260755@uaeh.edu.mx 
endulzante (de 30 a 600 veces) y un menor precio..$^{2-3}$ Por lo cual esta revisión tiene el objetivo de presentar la evidencia científica disponible en artículos científicos sobre el efecto que tienen las bebidas endulzadas con relación a la ingesta de energía y la acumulación del tejido adiposo. Se utilizaron las bases de datos de PubMed, Elsevier, Cochrane y SciELO, se incluyeron artículos publicados en el periodo de 2010-2020, sin embargo, cuando la información de otros artículos previos a la fecha indicada se consideró relevante, estos fueron incluidos.

\section{Hechos históricos de los endulzantes}

El primer endulzante artificial utilizado fue la sacarina, descubierta en 1879, la cual se utilizó principalmente con fines industriales. La sacarina de sodio es una sulfamida, forma sales y es aproximadamente 300 veces más dulce que el azúcar, aunque en altas concentraciones produce un gusto metálico. ${ }^{3}$

El jarabe de maíz de alta fructosa (JAMF) es un producto que se obtiene de la molienda del grano de maíz por medio de una triple hidrólisis ácida del almidón, por la acción de la enzima glucosa isomerasa. Así se obtiene el JMAF 42 y por medio de un intercambio iónico el JMAF 55; es un jarabe muy dulce, si consideramos el poder endulzante de la sacarosa como 1, el del JMAF 55 es de 1.3. ${ }^{2}$

La glucosa es el monosacárido más abundante en la naturaleza, es una molécula de 6 carbonos y es una aldosa, es un azúcar simple que se encuentra en forma libre en las frutas y en la miel. La industria alimentaria la denomina dextrosa, nombre derivado de la glucosa dextrorrotatoria. En comparación con la sacarosa, la glucosa tiene un dulzor de $0.75 .^{2}$

Tabla 1. Dulzor, utilización y características generales de algunos endulzantes

\begin{tabular}{|c|c|l|l|}
\hline $\begin{array}{c}\text { Endulzante } \\
\text { (Fórmula) }\end{array}$ & Dulzor & Características & \multicolumn{1}{|c|}{ IDA ${ }^{(4)}$} \\
\hline $\begin{array}{l}\text { Sacarosa } \\
\mathrm{C}_{12} \mathrm{H}_{22} \mathrm{O}_{11}\end{array}$ & 1.0 & $\begin{array}{l}\text { Disacárido, al } \\
\text { hidrolizarse se } \\
\text { obtiene una } \\
\text { molécula de } \\
\text { fructosa y otra } \\
\text { de glucosa. }\end{array}$ & $\begin{array}{l}5 \mathrm{mg} / \mathrm{kg} \text { de } \\
\text { peso } \\
\text { corporal por } \\
\text { día para los } \\
\text { adultos y } \\
\text { los niños. }\end{array}$ \\
\hline $\mathrm{JMAF}_{6} \mathrm{H}_{12} \mathrm{O}_{6}$ & 1.30 & $\begin{array}{l}\text { Es un jarabe } \\
\text { que se obtiene } \\
\text { de la digestión } \\
\text { del almidón de } \\
\text { maíz, las } \\
\text { glucosas } \\
\text { obtenidas se } \\
\text { someten a } \\
\text { isomerización }\end{array}$ & $\begin{array}{l}\text { No } \\
\text { establecido. }\end{array}$ \\
\hline
\end{tabular}

\begin{tabular}{|c|c|c|c|}
\hline & & $\begin{array}{l}\text { para obtener } \\
\text { fructosa. }\end{array}$ & \\
\hline $\begin{array}{l}\text { Dextrosa } \\
\mathrm{C}_{6} \mathrm{H}_{12} \mathrm{O}_{6}\end{array}$ & 0.75 & $\begin{array}{l}\text { La glucosa es } \\
\text { un } \\
\text { monosacárido, } \\
\text { se utiliza como } \\
\text { sustituto de } \\
\text { otros } \\
\text { endulzantes } \\
\text { como la } \\
\text { sacarosa. }\end{array}$ & $\begin{array}{l}\text { No } \\
\text { establecido. }\end{array}$ \\
\hline $\begin{array}{l}\text { Sucralosa } \\
\mathrm{C}_{12} \mathrm{H}_{19} \mathrm{C}_{13} \mathrm{O}_{8}\end{array}$ & $\begin{array}{l}\text { Aprox. } \\
600\end{array}$ & $\begin{array}{l}\text { La sucralosa es } \\
\text { muy estable y } \\
\text { es capaz de } \\
\text { retener su } \\
\text { dulzor cuando } \\
\text { se somete a } \\
\text { una elevada } \\
\text { temperatura y } \\
\text { acidez. }\end{array}$ & $\begin{array}{l}15 \mathrm{mg} / \mathrm{kg} \text { de } \\
\text { peso } \\
\text { corporal al } \\
\text { día. }\end{array}$ \\
\hline $\begin{array}{c}\text { Stevia } \\
\text { (Esteviol) } \\
\mathrm{C}_{20} \mathrm{H}_{30} \mathrm{O}_{3}\end{array}$ & $200-300$ & $\begin{array}{l}\text { La Stevia es un } \\
\text { compuesto } \\
\text { natural con alto } \\
\text { poder } \\
\text { endulzante. }\end{array}$ & $\begin{array}{l}4 \mathrm{mg} / \mathrm{kg} \\
\text { peso cor- } \\
\text { poral } \\
\text { (expresados } \\
\text { como } \\
\text { esteviol). }\end{array}$ \\
\hline
\end{tabular}

JMAF: jarabe de maíz de alta fructosa. IDA: ingesta diaria admisible.

La sucralosa es un endulzante artificial descubierto en 1976, que se obtiene por la halogenación selectiva de la molécula de sacarosa. Es entre 500 a 700 veces más dulce que el azúcar y se absorbe de forma limitada por el tracto gastrointestinal. ${ }^{3}$

La Stevia rebudiada es una planta selvática subtropical del alto Paraná, nativa del noroeste de la provincia de Misiones en Paraguay, donde era utilizada por los nativos como medicina curativa, llamada por las tribus de guaraníes como ka'a he'ê o "yerba dulce". ${ }^{3}$ El compuesto es 300 veces más dulce que la sacarosa. ${ }^{4}$ Las características y fórmulas de los endulzantes se presentan en la tabla 1.

\section{Dulzor y preferencia alimentaria}

El deseo humano por el sabor dulce es común en todas las edades, razas y culturas. El dulzor de un alimento influye en la nutrición humana, ya que permite seleccionar con más agrado aquellos alimentos que proporcionan energía (hidratos de carbono). Los bebés y los niños 
pequeños en particular seleccionan sus alimentos en relación con el sabor, prefiriendo el dulce. ${ }^{5}$ Se han descrito los mecanismos cerebrales que se activan tras la unión de una molécula dulce con su receptor en la boca (T1R2/T1R3). Estos receptores periféricos en un primer momento no distinguen entre endulzantes no calóricos y calóricos y basta con el estímulo del receptor para que se desencadene una cascada de procesos fisiológicos en la periferia y el cerebro, que además son placenteros para el individuo. ${ }^{6}$ Los receptores del gusto dulce se expresan también en el intestino y páncreas. ${ }^{6}$

Las investigaciones sugieren que estas preferencias son innatas y están presentes incluso antes del nacimiento. ${ }^{7}$ Muchos factores influyen en el desarrollo de las preferencias alimentarias en los niños, la primera está relacionada con los sabores de la dieta materna y después del nacimiento de la experiencia temprana con los sabores que ocurren en la leche humana. ${ }^{8}$ Los recién nacidos pueden diferenciar distintos grados de dulzor y consumirán un mayor volumen de una solución que tenga un sabor más dulce. Sus caras se relajan al degustar algo dulce, y esta relajación es a menudo acompañada de una sonrisa, esto debido a que los hidratos de carbono son censados como fuente de energía que además produce una sensación placentera. ${ }^{9}$

\section{Endulzantes y la regulación del apetito}

El apetito es regulado por distintos mecanismos entre los que se encuentran la estimulación de la fase cefálica la cual ocurre antes de que los alimentos ingresen al estómago, esta respuesta se desencadena por la vista, el pensamiento, el olor y el gusto; los dos primeros estimulan la corteza cerebral y los dos últimos el hipotálamo. ${ }^{10}$ Algunas investigaciones proponen la hipótesis de que la falta de activación de la respuesta en fase cefálica puede incrementar el riesgo de obesidad y, contrariamente, otras plantean que la activación de respuestas en fase cefálica, a partir de ingerir o simplemente con la exposición a alimentos dulces, pueden producir alteraciones en el consumo de alimentos. ${ }^{11}$

El estómago proporciona señales de apetito, basándose principalmente en el volumen que pueda estar o no cubierto del mismo, mientras que, por el contrario, el intestino es más sensible a las señales de la presencia de nutrientes, señales que dependen de osmorreceptores a nivel intestinal y quimiorreceptores a nivel gástrico. ${ }^{12}$ Aquellas bebidas que contienen EC, contienen más energía sobre una carga osmótica que puede ser igual o incluso menor a la producida por los ENC. A la misma osmolaridad, los EC inducen un vaciamiento más lento independientemente de los efectos osmóticos. ${ }^{12} \mathrm{La}$ activación de señales tanto a nivel intestinal como gástricas, a partir de la presencia de nutrientes, tiene efectos sinérgicos sobre la saciedad. ${ }^{12}$

Cada macronutriente estimula de forma diferenciada la liberación de péptidos a nivel del tubo digestivo. Los hidratos de carbono producen un estímulo para la secreción del péptido semejante al glucagón tipo 1 (GLP1), el cual regula la saciedad de forma semejante a la incretina. Se ha propuesto que los ENC no estimulan tal liberación de péptidos y por tanto se produciría una menor sensación de saciedad provocando un aumento del consumo energético. ${ }^{13}$ Evidencias recientes muestran que existen receptores con propiedades similares a los receptores del sabor dulce situados en la lengua, a nivel del tracto gastrointestinal que estimulan la liberación de GLP-1, lo que podría otorgar a los ENC algún papel en la regulación de dichos sistemas mediados por incretinas. ${ }^{13,14}$

El uso de endulzantes y sus compuestos metabólicos pueden alterar el funcionamiento óptimo de la microbiota intestinal y esto incrementar el riesgo de obesidad. ${ }^{15}$ Otros estudios han evidenciado que un ahorro/supresión de energía por la sustitución de hidratos de carbono con ENC podría provocar una sobre compensación en el consumo de energía, que llegue incluso a superar el déficit energético inducido por el endulzante, ${ }^{16}$ asociado a defectos en la señalización.

\section{Ingesta de endulzantes no calóricos y posibles riesgos a la salud}

Durante las últimas décadas el consumo de endulzantes y el riesgo de cáncer ha sido tema de diversos estudios, siendo la sacarina en ratas de laboratorio la que ha mostrado la evidencia más concluyente. Un metaanálisis pone en evidencia que 1 de 20 trabajos comprueba que altas dosis de sacarina en una generación de ratas aumenta significativamente las neoplasias (cáncer de vejiga) al ser comparada con los controles. ${ }^{17}$ Estos resultados no son reproducibles en humanos ya que el metabolismo de la sacarina en las ratas es distinto, en humanos la sacarina no produce litiasis, ya sea del tracto urinario o lesiones epiteliales. ${ }^{17}$

Un estudio realizado en ratas Sprague-Dawley (machos y hembras) tratadas con aspartamo (0 a 100000 ppm) y seguidas hasta su muerte natural, encontró en hembras un aumento de neoplasias, sin una respuesta dependiente de la dosis. ${ }^{18}$ En otro estudio realizado en 1010 casos de diferentes tipos de cáncer confirmado y 2107 controles, no se demostró asociación entre el consumo alimentos que contenían ENC (incluyendo el aspartamo) y el riesgo de cáncer. $^{19}$

Aunque los endulzantes bajos en calorías y no calóricos imparten dulzura a los alimentos y bebidas, se trata del 
único rasgo que tienen en común, ya que representan un grupo diverso de compuestos con diferencias importantes en su destino metabólico, ${ }^{20}$ y en los posibles efectos asociados a su consumo.

\section{Endulzantes calóricos y no calóricos en la ganancia de peso}

Existe controversia si el consumo de ENC incrementa el riesgo de obesidad, se ha observado que la ingesta de sacarosa provoca un aumento de la glucosa plasmática y un vaciamiento gástrico más lento en comparación con la sucralosa, que no provoca cambios en los niveles de glucosa plasmática, al no estimular la liberación de insulina y de incretinas, contrario a lo que no sucede con sacarosa. ${ }^{21}$

Por otro lado, se discute que los ENC no poseen un poder saciante como los EC, inclusive podrían causar la sensación de hambre estimulando a comer en exceso, además podrían estimular los receptores del gusto, creando adicción al sabor dulce. ${ }^{22}$

Respecto a las respuestas fisiológicas de los endulzantes se ha demostrado que tras su consumo se incrementa la expresión de los transportadores intestinales de glucosa (SGLT1) y la inducción de la translocación de los transportadores de glucosa (GLUT2) en el borde en cepillo de la membrana del epitelio intestinal; el aumento de dichos cambios puede facilitar la absorción y el metabolismo de los azúcares ingeridos. Sin embargo, en el caso de los ENC, debido a que estos cambios intestinales no están acompañados por la presencia de azúcares, en respuesta se podría aumentar el consumo de energía. ${ }^{23}$

Estudios en seres humanos han documentado la activación neuronal diferencial en el hipotálamo durante el consumo de EC en comparación con los ENC. En conjunto, estos datos apoyan la hipótesis de que la señalización de sabores dulces en la ausencia de calorías produce efectos significativamente diferentes en comparación con el consumo de los sabores dulces asociados con las calorías, y con el tiempo estos efectos pueden contribuir a un balance energético positivo y el aumento de peso corporal. ${ }^{2}$

A todo anterior se debe considerar que existe una amplia gama de estructuras de hidratos de carbono disponibles para el mercado de alimentos y las diferentes propiedades fisicoquímicas que poseen, lo que hace necesario identificar los efectos de la estructura de los hidratos de carbono y los ENC sobre las señales de saciedad pre absortiva y absortiva, así como en la ganancia de peso corporal. ${ }^{24}$

Se ha descrito que los niños que consumen más EC tienen mayor probabilidad de tener sobrepeso que los que tienen un consumo limitado. ${ }^{20,} 25$ El consumo de ENC (sucralosa, aspartame, Stevia, ciclamato) se asocia con el aumento de índice de masa corporal (IMC) y grasa corporal en la población infantil, ${ }^{26}$ siendo mayor la ganancia de peso en aquellos que lo consumen diariamente en comparación con un consumo semanal. ${ }^{27}$ Existe un mayor aumento de peso en animales que consumen sacarina en comparación con sacarosa, esto fue probado en un tratamiento de 16 semanas con ratas Wistar macho, donde se concluye que a largo plazo la sacarina tiene un efecto positivo en el peso corporal. ${ }^{28}$ Existe controversia en los efectos de otros ENC ya que, en otro estudio donde se probó el efecto de sucralosa y aspartame no se encontraron diferencias en el aumento de peso corporal en ratas macho Sprague-Dawley con el grupo control. ${ }^{29}$ En otros trabajos se ha reportado que hay un mayor consumo de energía proveniente del alimento en los animales que consumen sacarina y acesulfame potásico en comparación de los que consumen glucosa. ${ }^{30}$

\section{Efectos metabólicos de los azúcares añadidos}

La liberación de calorías de azúcares añadidos requiere nutrientes como la tiamina, riboflavina y niacina para la formación de coenzimas intermediarias en la producción de ATP. ${ }^{31}$ Los hidratos de carbono que han sido aislados de su alimento natural, carecen de nutrientes y fibra, lo que podría estar produciendo una mayor carga metabólica en el cuerpo, en particular cuando contienen hidratos de carbono adicionales en forma de azúcares agregados, como pueden ser encontrados en las bebidas gaseosas, pastelillos, galletas, barras de pan, cereales de desayuno, granola, etc., a partir de los granos refinados con azúcares adicionales y endulzantes añadidos como conservantes y para mayor palatabilidad. ${ }^{32}$

Paradójicamente se ha descrito que en la obesidad existe un déficit de nutrientes y energía, lo que puede conducir a una menor movilización y oxidación de ácidos grasos del tejido adiposo. ${ }^{33}$ El déficit de nutrientes y energía está relacionado al consumo excesivo de bebidas endulzadas, lo que desplaza la ingesta de otros nutrientes como vitaminas y minerales. ${ }^{33}$

El consumo de azúcares añadidos altera la función de la mitocondria y afecta la generación de energía, esto puede dar lugar a una especie de "inanición interna" mediada por leptina y la resistencia a la insulina, lo que promueve señalización de hambre. ${ }^{34}$ Es por eso que los azúcares añadidos agotan la energía del cuerpo ya sea de las reservas de nutrientes de los tejidos o los obtenidos por los alimentos ingeridos. ${ }^{34}$

En conclusión, los azúcares añadidos desplazan a los alimentos con mejor calidad nutricional de la dieta y al 
mismo tiempo, aumentan las necesidades nutricionales, específicamente de vitaminas como la tiamina, la riboflavina y niacina; las cuales son necesarias para la oxidación de la glucosa, además, los fosfatos necesarios para la síntesis de ATP son utilizados para metabolizar la fructosa, lo que conduce al agotamiento del ATP celular. ${ }^{33}$ El azúcar también tiene la capacidad de alterar el apetito por alimentos de alta densidad energética, causando un mayor agotamiento de nutrientes. Esto es probablemente causado por la elevación de los niveles de insulina provocada por hidratos de carbono refinados, lo que puede conducir a presentar ansiedad por más hidratos de carbono, debido a una resistencia a la insulina que modifica la utilización de estos e inhibe la generación de energía a partir de ácidos grasos. ${ }^{35}$

En esencia, las células están ávidas de energía debido a que está inhibida la oxidación de ácidos grasos por la resistencia a la insulina producida por el sobreconsumo de bebidas endulzadas.

\section{Efectos del consumo de endulzantes calóricos y no calóricos en el tejido adiposo}

El tejido adiposo blanco tiene funciones endocrinas y desempeña un papel en la regulación de la homeostasis energética y sensibilidad a la insulina, se caracteriza por su capacidad para adaptarse y expandirse en respuesta a la energía excedente a través de procesos de la hipertrofia de los adipocitos y/o el reclutamiento y la proliferación de las células precursoras en combinación con la irrigación vascular y remodelación de la matriz extracelular. ${ }^{36}$ Sin embargo, en el contexto de la obesidad crónica sufre fibroinflamación, lo que compromete su funcionalidad y contribuye a aumento del riesgo de diabetes tipo 2 y enfermedades cardiovasculares. ${ }^{36,37}$

El exceso de adiposidad abdominal se correlaciona con alteraciones metabólicas y cardiovasculares secundarias a la obesidad. ${ }^{38}$ Lo anterior se debe a que los adipocitos intraabdominales tienen una actividad lipolítica más elevada, de tal forma que promueve la movilización de ácidos grasos al hígado, el cual responde aumentando la tasa de síntesis de triglicéridos y lipoproteínas de muy baja densidad (VLDL), desencadenando resistencia a la insulina, y como consecuencia un hiperinsulinismo. ${ }^{(39)}$ A medida que el adipocito crece y aumentan sus depósitos de triglicéridos, aumenta también la secreción de sustancias que inhiben la diferenciación de preadipocitos a adipocitos, dificultando así la entrada de triglicéridos a su interior y facilitando la salida de éstos hacia la circulación por medio de la lipólisis. A su vez el adipocito secreta varias proteínas llamadas adipocinas las cuales tienen acción sobre múltiples sistemas regulando diversos procesos metabólicos. ${ }^{40}$ Una acción esencial de las adipocinas es su participación en la respuesta de las células a la insulina, favoreciendo la utilización de glucosa y el metabolismo de los ácidos grasos. La leptina y la adiponectina favorecen la acción de la insulina, y por otro lado, la resistina, el factor de necrosis tumoral a y la interleucina- 6 interfieren con ella, viniendo a formar parte del proceso de resistencia a la insulina. ${ }^{40}$

El consumo de endulzantes calóricos y no calóricos se ha relacionado con la plasticidad del tejido adiposo. Existe evidencia reciente en animales del efecto de algunos ENC como la sucralosa o steviol en el peso y la grasa corporal, se ha mostrado que no existen diferencias en comparación con animales alimentados con agua. ${ }^{41}$

En contraparte los EC tienen una mayor evidencia del consumo y el desarrollo de esteatosis hepática; este es el caso del JMAF. ${ }^{42}$ En animales de experimentación, como ratones, perros y primates, es conocido que dietas altas en energía a partir de fructosa o sacarosa inducen a la hiperlipidemia e hipertensión, lo cual eventualmente se asociaría con aumento del riesgo de enfermedades cardiovasculares. ${ }^{42}$

La glucosa estimula una mayor captación del disacárido por el tejido adiposo mediado por la epinefrina en contraste con su acción en el músculo. ${ }^{43}$ Sin embargo, el aumento de la captación de glucosa causado por la adrenalina se acompaña un aumento en la liberación de ácidos grasos libres. ${ }^{44}$

Existe una relación entre el sitio de almacenamiento de tejido adiposo y el tipo de EC consumido, en el caso de la fructosa se incrementa la acumulación de tejido adiposo subcutáneo, visceral y esquelético. ${ }^{45}$ En otros estudios realizados con sacarosa y glucosa se han encontrado efectos similares en la acumulación de grasa en órganos sin especificar donde se almacena más masa grasa. ${ }^{46} \mathrm{De}$ los demás tipos de ENC (sucralosa, Stevia) en humanos no se han encontrado evidencias científicas en las cuales se demuestre un mayor almacenamiento de tejido adiposo en zonas específicas. ${ }^{47}$

Debido a que el sobrepeso y la obesidad son un problema de salud pública en México y que esto está relacionado con el consumo de bebidas endulzadas se sabe que los ENC producen una menor sensación de saciedad y por ende provocan un aumento del consumo energético, creando adicción por este sabor dulce y finalmente un aumento de peso; por lo cual es importante tener un consumo moderado y limitado de los EC y ENC, pues como se ha registrado en la evidencia existe una mayor ganancia de peso en los participantes que los consumen diariamente en comparación a aquellos con un consumo semanal. Al mismo tiempo algunos estudios demuestran que estos ENC (sacarina, aspartamo) se asocian con el riesgo de padecer cáncer. 
Otro punto importante a tratar es que los azucares añadidos desplazan a los alimentos con mejor calidad nutricional de la dieta, alteran el apetito por alimentos de alta densidad energética y causan un agotamiento de nutrientes.

Asimismo, el aumento de la adiposidad se ve relacionado con alteraciones metabólicas y cardiovasculares secundarias a la obesidad, aumentando los niveles de triglicéridos, glucosa, LDL, etc. circulantes y de acuerdo a algunos estudios se observa que hay una relación entre el EC y ENC consumido y su sitio de acumulación. Es evidente que existe un efecto en la regulación de la ingesta de energía y almacenamiento de tejido adiposo asociados con el tipo de endulzante consumido, por lo cual, no todos EC o ENC deben evaluarse de la misma forma.

Debido a esto es importante la evaluación de forma aislada de cada uno de estos endulzantes que son tan usados en la industria alimentaria y así estar más informados sobre el riesgo o limitaciones a tener de cada uno de ellos bajo evidencia científica.

\section{Referencias}

1. Durán S, Cordón K, Rodríguez M. Edulcorantes no nutritivos, riesgos, apetito y ganancia de peso. Rev Chil Nutr. 2013;40(3):309-14.

2. Durán S. Edulcorantes no nutritivos, riesgos y ganancia de peso. Universidad de San Carlos de Guatemala, Guatemala. [Science and Health ]. 2015; Vol 40:10-2.

3. Rodríguez MP. Edulcorantes no nutritivos, riesgos y ganancia de peso. Universidad de San Carlos de Guatemala, Guatemala. [Science and Health ]. 2015; Vol 40:20-2.

4. Organización Mundial de la Salud. Carbohydrates in Human Nutrition, 1998.

5. Murray RD. Savoring Sweet: Sugars in Infant and Toddler Feeding. Annals of nutrition \& metabolism. [Review Research Support, NonU.S. Gov't]. 2017;70 Suppl 3:38-46.

6. Harris R. Effect of repeated stress on body weight and body composition of rats fed low and high fat diets. American Physiology society. 1998;44:1928-38.

7. Joseph-Bravo P. El estrés y sus efectos en el metabolismo y el aprendizaje. Biotecnology. 2007;14:65-76

8. Katz R. Acute chronic stress effects on open field activity in the rat: Implications for a model of depression. Neuroscience Biobehavorial. $1991 ; 5(247-251)$

9. Renwick A. Edulcorantes bajos en calorías: funciones y beneficios International Sweeteners Association. 2012:1-31.

10. Agúero S. Consuption of non nutritive sweeteners and nutritional status in humans. . San Sebastian's University. [Science and Health ]. 2014; Vol 1:Page 5.

11. Mattes R. Nonnutritive sweetener consumption in humans: effects on appetite and food intake and their putative mechanisms. Am J Clin Nutr. [Science ]. 2009; Vol 1:1-14.
12. Bourne C. Non-nutritive sweeteners, energy balance and glucose homeostasis. Curr Opin Clin Nutr Metab Care. [Science ]. 2011; Vol 4 14.

13. Gardner C. American Heart Association Nutrition Committee of the Council on Nutrition, Physical Activity and Metabolism, Council on Arteriosclerosis, Thrombosis and Vascular Biology, Council on Cardiovascular Disease in the Young; American Diabetes Association. Nonnutritive sweeteners: current use and health perspectives: a scientific statement from the American Heart Association and the American Diabetes Association. Diabetes Care. 2012; vol 8:25-7.

14. Yang Q. Gain weight by "going diet?" Artificial sweeteners and the neurobiology of sugar cravings: Neuroscience. Yale J Biol Med. [Science ]. 2010; Vol 2 (101-8):83.

15. Torresani M. Manejo y consumo de productos dietéticos y edulcorantes no nutritivos. Revista Española de Nutrición Comunitaria 2007.

16. Pepino M. Non-nutritive sweeteners, energy balance and glucose homeostasis. Curr Opin Clin Nutr Metab Care. [Science]. 2011; Vol 4(391-5.):page 15

17. García A, Alemán J. Una visión global y actual de los edulcorantes, aspectos de regulación. Nutrición Hospitalaria. [Food and Health ]. 2013; Vol 28 num 4 17-22.

18. Belpoggi F SM, Padovani M, Degli Esposti D, Lauriola M, Minardi F. Results of long-term cercinogenicity bioassay on Sprague-Dawley rats exposed to aspartame administered in feed. . Ann NY Acad Sci. 2006(1076:559-77. ).

19. Magnuson BA BG, Doull J. Aspartame: a safety evaluation based on current use levels, regulations, and toxicological and epidemiological studies. . Crit Rev Toxicol. 2007(37: 629-727. ).

20. Mattes R. Nutritively sweetened beverage consumption and body weight: a systematic review and meta-analysis of randomized experiments. Public Health Nutr. 2011;12:46-65.

21. Carbajal A. Manual de Nutrición y Salud Pública. Métodos, bases científicas y aplicaciones. [Science]. 2013;Vol 3 22-30.

22. García Almeida. Una visión global y actual de los edulcorantes. Aspectos de regulación. Nutrición Hospitalaria. 2013; Vol 28 núm 4:1731.

23. Casado Fdez. Una visión global y actual de los edulcorantes. Aspectos de regulación. Nutrición Hospitalaria. [Science]. 2013; Vol 28 núm 4:17-31

24. Brown J. Artificial sweeteners: a systematic review of metabolic effects in youth. Int J Pediatr Obes. 2010;5:10-2

25. Robin M. Do non-nutritive sweeteners influence acute glucose homeostasis in humans? A systematic review Physiology \& Behavior. 2017:17-26

26. TeMorenga L. Dietary sugar sand body weight: systematic review andmeta-analyses of randomised controlled trials and cohorts tudies BMJ. 2013:1-25.

27. Bantle J. Metabolic effects of dietary fructose in diabetic subjects. Diabetes Care. 2012;15:68-76.

28. Jiantao M. Potential link between excess added sugar intake and ectopic fat: a systematic review of randomized controlled trials. Nutrition Reviews. 2012;vol. 74:18-32.

29. Johnston R. No difference between highfructose and high-glucose diets on liver triacylglycerol or biochemistry in healthy overweight. Gastroenterology. 2013;145:1016-25.

30. Behall K. Effect of starch structure on glucose and insulin responses in adults. . Am J Clin Nutr. [Science and Health]. 1998;47:428-32.

31. Guthrie J.F. MJF. Food sources of added sweeteners in the diets of Americans. J Am Diet Assoc. [Science and Health ]. 2000:43-8. 
32. Logue C. The potential application of a biomarker approach for the investigation of low-calorie sweetener exposure. Proc Nutr Soc 2. [Science and Health ]. 2016:216.

33. Anderson G. The use of low-calorie sweeteners by adults: impact on weight management. J Nutr. 2012;6:3-9

34. Bonacchi K. Sucrose taste but not polycose taste conditions flavor preferences in rats. Physiology \& Behavior. 2008;1-2:235-44.

35. Ashleigh E. Early Exposure to Nonnutritive Sweeteners and Long-term Metabolic Health: A Systematic Review Physiology \& Behavior. 2017;137(1-12)

36. Cushman S. Structure-function relatioship in the adipose cell. . JCell Biol. [Science ]. 2010; Vol 1:page 15.

37. Gartner L. Histología. Texto y atlas. McGraw-Hill Interamericana. [Science]. 2008; $3^{\mathrm{a}}$ edición:page 22

38. Geneser F. Histology. Editorial Medica Panamericana. [Science ]. 2010;3 $3^{\text {a }}$ edición:page 33.

39. Arner E. Adipocyte Turnover: Relevance to Human Adipose Tissue Morphology. American Diabetes Assosiation. [Science and Health ] 2010;1:105-9.

40. Hocking S. Subcutaneous fat transplantation alleviates diet-induced glucose intolerance and inflammation in mice. Dietology. [Science and Health ]. 2015; vol 2:56-9.

41. Swithers S. High-intensity sweeteners and energy balance. . Physiol Behav. 2010;1:55-72.

42. Magee L. Sugar and artificially sweetened beverage consumption and adiposy changes: National longitudinal study. International Journal of Behavioral Nutrition ang Physical Activity. 2015;12:137.

43. Kleiman S. Can beverage companies cut calories while maintaining profits? . Journal of the International Association for the Study of Obesity. 2012;13:58-74.

44. Davidson T. General and persistent effects of high-intensity sweeteners on body weight gain and caloric compensation in rats. American Phychological Association. 2009;7:2-80

45. Riveros M. Consumo de fructosa y sus implicaciones para la salud malabsorción de fructosa e hígado graso no alcohólico. Nutrición Hospitalaria. 2014;29(491-499).

46. Douard V. Regulation of the fructose transporter GLUT5 in health and disease. Am J Physiol Endocrinol Metab. 2008;295:27-37.

47. Tovar A. The effect of moderate consumption of non-nutritive sweeteners on glucose tolerance and body composition in rat Department of Kinesiology and Nutrition Sciences. 2010:1-11. 